\title{
Assessment of SAPT(DFT) with meta-GGA functionals
}

\author{
Michał Hapka ${ }^{1,2}$ (D) $\cdot$ Marcin Modrzejewski ${ }^{2}$ (D) $\cdot$ Grzegorz Chałasiński $^{2}$ (D) $\cdot$ Małgorzata M. Szczęśniak $^{3}$ (I)
}

Received: 20 November 2019 / Accepted: 4 March 2020 / Published online: 15 April 2020

(C) The Author(s) 2020

\begin{abstract}
This work examines the suitability of meta-GGA functionals for symmetry-adapted perturbation theory (SAPT) calculations. The assessment is based on the term-by-term comparison with the benchmark SAPT variant based on coupled-cluster singles and doubles description of monomers, SAPT(CCSD). Testing systems include molecular complexes ranging from strong to weak and the He dimer. The following nonempirical meta-GGAs are examined: TPSS, revTPSS, MVS, SCAN, and SCAN0 with and without the asymptotic correction (AC) of the exchange-correlation potential. One range-separated meta-GGA functional, LCPBETPSS, is also included. The AC-corrected pure meta-GGAs (with the exception of MVS) represent a definite progress in SAPT(DFT) compared to pure GGA, such as PBEAC, with their more consistent predictions of energy components. However, none of the meta-GGAs is better than the hybrid GGA approach SAPT(PBE0AC). The SAPT(DFT) electrostatic energy offers the most sensitive probe of the quality of the underlying DFT density. Both SCAN- and TPSS-based electrostatic energies agree with reference to within 5\% or better which is an excellent result. We find that SCAN0 can be used in SAPT without the AC correction. The long-range corrected LC-PBETPSS is a reliable performer both for the components and total interaction energies.
\end{abstract}

Keywords Symmetry-adapted perturbation theory · SAPT(DFT) - Intermolecular interactions · Meta-GGA · Dispersion energy · Electrostatic energy $\cdot$ Exchange repulsion $\cdot$ DFT-SAPT

\section{Introduction}

Symmetry-adapted perturbation theory (SAPT) is an effective means of computing the interaction energies of non-covalent interactions with direct insights into their composition [1]. Non-covalent interactions result from a delicate balance of the attractive and repulsive components some of which, e.g.,

This paper belongs to the Topical Collection Zdzislaw Latajka 70th Birthday Festschrift

Electronic supplementary material The online version of this article (https://doi.org/10.1007/s00894-020-4340-9) contains supplementary material, which is available to authorized users.

Michał Hapka

hapka@ tiger.chem.uw.edu.pl

Małgorzata M. Szczęśniak

bryant@oakland.edu

1 Department of Chemistry, University of Michigan, Ann Arbor, MI 48109, USA

2 Faculty of Chemistry, University of Warsaw, Pasteura 1, 02-093 Warszawa, Poland

3 Department of Chemistry, Oakland University, Rochester, MI 48309, USA the dispersion energy, have no classical correspondent. The repulsive contributions are equally important and, with the exception of electrostatics, also nonclassical in nature because they originate from the antisymmetry requirement [2]. The only rigorous way to obtain the dispersion interactions, as well as the exchange contributions (in a weak symmetry forcing scheme), is via the application of SAPT methodology [3]. In its first many-electron formulation SAPT (the so called manybody, MB-SAPT) was a double perturbation theory expansion with respect to two perturbations: intermolecular interaction operator and the intramonomer correlation operator $[1,4]$. In this theory, many terms are needed to correctly describe particular interaction energy term. For example, the exact description of the electrostatic energy requires an infinite expansion in terms of intramonomer correlation [5, 6]. By contrast, if the monomers were already correlated, the electrostatic effect, as well as any other contribution, would be more straightforward to compute and interpret.

Advantages due to bypassing the double perturbation expansion have been explored in notable recent SAPT extensions. Holzer and Klopper developed SAPT based on quasiparticle energies and response functions from the GW method [7]. Boese and Jansen used accurate densities to construct Kohn-Sham (KS) exchange-correlation potentials for use in 
SAPT [8]. Korona employed CCSD density matrices and response functions in the formulation of SAPT(CCSD) which also bypasses double-perturbation theory [9]. Finally, Hapka et al. investigated the extent to which the complete active space monomers' wave functions recover the intrasystem correlation effects on the dispersion energy $[10,11]$.

If the density functional theory (DFT) were to be used for describing the monomer wave functions, the intrasystem correlation effects can be captured (at least in principle) giving rise to the simplest way of bypassing double perturbation SAPT expansion. This idea was independently developed by the groups of Szalewicz [5, 12-14] and Jansen [15-18] and known as SAPT(DFT) (or DFT-SAPT). The promise of this theory lies in the fact that if we had the accurate density functional and its derivatives, the theory would be exact to the second-order as far as the polarization terms are concerned. The exchange terms require one- and two-particle density matrices which cannot in principle be reproduced even with the exact KS determinant. In practice, the asymptotic form of the first-order exchange should be accurate in SAPT(DFT) [14]. Furthermore, the numerical evidence shows the excellent agreement between first- and second-order exchange terms and the available benchmarks [9, 14].

A path toward further improvement of SAPT(DFT) is to step-up onto a higher rung of the Jacob's ladder [19], i.e., to seek DFT functionals that are closer to the ideal of the accurate functional. To date, mainly GGA functionals and their hybrids have been employed in SAPT. As far as meta-GGAs are concerned, only Minnesota M05 [20] and M06 [21] functionals and their hybrids were examined in Ref. [9] with mixed results. Namely, M05 functional yielded decent electrostatic and dispersion components whereas in M06 these terms were exceptionally poor. The heavily parametrized nature of these functionals makes it difficult to ascertain what was the reason behind this disparity.

In the present paper, we intend to systematically examine a newer group of nonempirical meta-GGAs for their suitability in SAPT(DFT). Meta-GGA exchange-correlation energies depend on kinetic energy density, $\tau$, in addition to density and its gradient as GGAs do. This gives them more functional flexibility to satisfy a larger number of exact constraints. For example, at the GGA level, it is impossible to detect one-electron densities, e.g., hydrogen atom density, which leads to the one-electron self-interaction error. It is only the orbital-dependent $\tau$ which offers this capability. Nonempirical meta-GGA functionals are constructed to satisfy the constraints that the exact functional is known to obey. The latest fruit of this effort led by the Perdew, Ruzsinszky, and Sun team is the functional SCAN (strongly constrained appropriately normed) which satisfies all 17 known exact constraints (among which there are, for example, tighter lower bound for exchange, gradient expansion accurate to 4th order, one-electron self-correlation equal zero, etc.) [22]. Newer meta-GGAs, such as MVS [23] and SCAN, reputably distinguish between paradigm bonding cases in chemistry and physics: covalent, metallic, and noncovalent. This is because of the skillful incorporation of kinetic energy density in the form of the nondimensional kinetic ingredient: $\alpha=\left(\tau-\tau^{\mathrm{W}}\right) / \tau^{\mathrm{UEG}}$ where $\tau^{\mathrm{W}}$ is von Weizsacker kinetic energy density of one-electron system and $\tau^{\mathrm{UEG}}$ stands for the kinetic energy density of the uniform electron gas. $\alpha$ differs for covalent, metallic, and noncovalent bonds. It is interesting if this ability reflects on density functional's performance in SAPT. Another way of incorporating meta-GGA ingredients is through the range-separation procedure build on the Becke-Roussel exchange hole [24] that includes both $\tau$ and the Laplacian of density. One such rangeseparated meta-GGAs is tested here in SAPT as well. The results for meta-GGAs will be compared with the current SAPT(DFT) "standard-bearer" GGAs, PBEAC, and PBE0AC, gauging the results against SAPT(CCSD) benchmarks [9]. To complete the comparison, we also employ the wave function SAPT; the details of which will be described in the next section.

\section{Computational details}

The framework for evaluation of new functionals' performance is a term-by-term comparison with SAPT(CCSD) of Korona [9] used as reference. The test set includes the same dimers as in Ref. [9]. The set of molecules ranges in strength of interactions from very strong hydrogen bonds (FHF) ${ }^{-}$, where proton is shared, to very weak van der Waals complexes involving $\mathrm{He}$ bound to a molecule, e.g., $\mathrm{CO}_{2}$. It also includes third period elements. Following Ref. [9] two subsets are considered. Larger set $\mathrm{S}_{1}$ containing 21 dimers was tested in the smaller aug-cc-pVDZ basis set $[25,26]$. The $S_{2}$ set is its subset from which six large dimers were removed. The $\mathrm{S}_{2}$ set was tested in the larger, aug-cc-pVTZ, basis set. This choice is dictated by the high computational demands of SAPT(CCSD) reference calculations. Compared to Ref. [9], we also computed methane dimer SAPT(CCSD) benchmark data in aug-ccpVTZ basis set and included them in the set $\mathrm{S}_{2}$. Both sets are referred to as TK21. Another important paradigm is $\mathrm{He}_{2}$. Here, we conduct a similar term-by-term comparison as in the work of Cencek and Szalewicz [27, 28] thus extending their insights to meta-GGAs: pure, hybrid, and rangeseparated hybrid. The details including the basis set, geometry, and the reference values are the same as in their work.

The following functionals are included in the present study: TPSS [29], revTPSS [30], MVS [23], SCAN [22], and SCAN0 [31] representing a progression in the meta-GGA design. Range-separated meta-GGAs are still quite rare. We include one such functional, LC-PBETPSS, designed using the range-separation method proposed in Ref. [32]. In this method, the short-range exchange is obtained using BeckeRoussel exchange hole which is exact for hydrogenic systems and contains in its formulation the meta-GGA ingredients. If 
combined with PBE exchange, this method elevates PBE to the meta rung. Such a meta-GGA exchange is then combined with the TPSS correlation thus forming the LC-PBETPSS meta-GGA functional. If range-separated functionals are to be used in SAPT, the range-separation parameters should be optimized so as to minimize the difference, $\Delta_{\mathrm{XC}}$, between the vertical ionization potential, $I P$, and the negative energy of the highest occupied molecular orbital (HOMO), $\mathrm{e}_{\text {HOMO }}$, the method known as the IP tuning. In this work, we used the monomer range-separation parameters previously optimized for LC- $\omega$ PBE (i.e., for range-separated GGA) from Ref. [33].

Range-separated hybrids employ $\mathrm{v}_{\mathrm{XC}}$ potentials which converge to the correct $-1 / \mathrm{r}$ asymptote [34]. This is not the case for the other meta-GGAs and their hybrids. To remedy this problem, $\mathrm{V}_{\mathrm{XC}}$ potentials are equipped with the so called asymptotic correction (AC) which ensures that the correct $\mathrm{v}_{\mathrm{XC}} \rightarrow-1 / \mathrm{r}+\Delta_{\mathrm{XC}}$ limit is reached. In the present work, the gradient-regulated asymptotic correction (GRAC) [35] is employed and applied to TPSS, revTPSS, MVS, SCAN, and SCAN0 functionals. To accommodate the asymptotic correction to meta-GGAs, we have supplied the existing GGA implementation with the kinetic energy derivatives scaled by (1- $\mathrm{fRAC}^{\mathrm{GR}}$ ), where $\mathrm{f}^{\mathrm{GRAC}}$ is the GRAC interpolation factor $[35,36]$. For practical reasons, we have omitted the extra terms described in Refs. [27, 36]. The monomer ionization potentials needed for the correction are taken from experiment for consistency with results of Ref. [9].

The SAPT(DFT) theory represents the interaction energy $E_{\text {int }}$ as

$E_{\mathrm{int}}=E_{\mathrm{elst}}^{(1)}+E_{\mathrm{exch}}^{(1)}+E_{\text {ind }}^{(2)}+E_{\text {exch-ind }}^{(2)}+E_{\text {disp }}^{(2)}+E_{\text {exch-disp }}^{(2)}$.

All exchange energies are evaluated in the $\mathrm{S}^{2}$ approximation (S refers to the overlap integral) to permit the direct comparison with the SAPT(CCSD) benchmarks using this approximation. The second-order terms $E_{\text {ind }}^{(2)}$ and $E_{\text {disp }}^{(2)}$, and their respective exchange counterparts, are obtained from the coupled Kohn-Sham (KS) density-response functions with the underlying pure ALDA (adiabatic local density) approximation or hybrid kernels, depending on the type of functional. All calculations were performed with the internally modified Molpro 2012 program suite [37] with no density fitting.

The MVS, SCAN, and SCAN0 computations involved an extra dense $\log 3$ radial grid $\left(n_{r}=250\right)$ (from Ref. [38]).

The wave function SAPT (MB-SAPT) is also included in the comparison. The MB-SAPT version, denoted SAPT2+ (CCD), includes the following elements:

$$
\begin{aligned}
E_{\mathrm{int}}^{\mathrm{SAPT} 2+(\mathrm{CCD})}= & E_{\text {elst }}^{(10)}+E_{\mathrm{elst}, \text { resp }}^{(12)}+E_{\text {exch }}^{(10)}+E_{\text {exch }}^{(11)}+E_{\text {exch }}^{(12)} \\
& +E_{\text {ind,resp }}^{(20)}+{ }^{t} E_{\text {ind }}^{(22)}+E_{\text {exch-ind,resp }}^{(20)} \\
& +{ }^{t} E_{\text {exch-ind }}^{(22)}+E_{\text {disp }, \mathrm{CCD}}^{(2)}+E_{\text {exch-disp }}^{(20)} .
\end{aligned}
$$

All the components except for the dispersion energy are the same as in the SAPT2 variant, as described by Hohenstein and Sherrill [39]. The second-order dispersion term $E_{\text {disp,CCD }}^{(2)}$ is calculated at the CCD + ST (CCD) level of theory as first proposed by Williams et al. [40]. The total interaction energy excludes the so called $\delta_{\mathrm{HF}}$ term (see, e.g., [41]). The SAPT2+ (CCD) calculations were performed using Psi4 program [42] with the use of natural orbital truncation techniques for the $\mathrm{CCD}+\mathrm{ST}(\mathrm{CCD})$ dispersion energy [43]. The wave function SAPT denoted SAPT(HF) involves all the terms computed analogously to Eq. (1) except for employing the HartreeFock (HF) wave function instead of KS. Note that in SAPT(HF), the exchange-dispersion energy is obtained at the coupled level of theory, whereas in SAPT2 + (CCD), this component is included within the uncoupled approximation.

The relative percent errors in energies $\left(\mathrm{E}_{\mathrm{i}}\right)$ with respect to reference values $\left(\mathrm{E}_{\mathrm{i}, \mathrm{ref}}\right)$ shown in the plots and in the tables below are defined as:

$\Delta(\%)=\left(\mathrm{E}_{\mathrm{i}}-\mathrm{E}_{\mathrm{i}, \text { ref }}\right) /\left|\mathrm{E}_{\mathrm{i}, \mathrm{ref}}\right| \times 100$.

\section{Results and discussion}

\section{a. $\mathrm{He}_{2}$ dimer}

The analysis begins with the interaction energy components of the He dimer shown in Table 1. The previous SAPT(DFT) suitability study of this type by Cencek and Szalewicz [27] involved only GGAs and their hybrids. It is here extended to include SCAN, SCAN0 with AC-corrected variants, as well as LC-PBETPSS, a range separated metaGGA. As seen in Table 1, SAPT(DFT) based on SCAN represents a dramatic improvement with respect to PBE. The errors in the first-order terms are reduced by one half, whereas those in the induction and exchange-induction are reduced by nearly an order of magnitude. The dispersion energy in SCAN is only $4 \%$ away from the reference. When compared with PBE0, the SCAN components are considerably closer to the reference values. This observation goes against the conventional wisdom which assumes that SCAN is comparable in performance to hybrid GGAs. Hybrid SCAN0 affords further improvements in energy components particularly for dispersion energy. Adding the AC correction brings the expected improvement to both GGA and meta-GGAs' results. Although individual SCANAC and SCANOAC components exhibit larger errors compared to PBE0AC, the total interaction energies are in better agreement with the Gaussian geminal reference. This results from the error compensation due to systematic underestimation of all the energy contributions.

The range-separated meta-GGA LC-PBETPSS yields both the components and the total interaction energy in close 
Table 1 Percent errors in $\mathrm{He}_{2}$ with respect to the reference ${ }^{\mathrm{a}}$ for GGA and meta-GGA functionals in SAPT (DFT) at equilibrium separation of 5.6 bohr. All calculations were performed in d-aug-cc-pV5Z + mid-bond functions (the same as in Ref. [28]). Units are $\mathrm{cm}^{-1}$

\begin{tabular}{|c|c|c|c|c|c|c|c|}
\hline Method & $E_{\text {elst }}^{(1)}$ & $E_{\mathrm{exch}}^{(1)}$ & $E_{\text {ind }}^{(2)}$ & $E_{\mathrm{exch}-\mathrm{ind}}^{(2)}$ & $E_{\text {disp }}^{(2)}$ & $E_{\text {exch-disp }}^{(2)}$ & $E_{\text {int }}$ \\
\hline Reference values ${ }^{\mathrm{a}}$ & -1.187 & 8.54 & -0.196 & 0.177 & -15.565 & 0.515 & -7.716 \\
\hline PBE & -101.7 & 118.0 & -156.5 & 165.9 & -26.0 & 85.4 & 68.0 \\
\hline SCAN & -48.4 & 60.3 & -19.2 & 20.9 & -4.4 & 18.5 & 51.7 \\
\hline PBE0 & -55.9 & 65.2 & -83.5 & 83.0 & -14.2 & 46.5 & 37.8 \\
\hline SCAN0 & -27.6 & 35.1 & -15.6 & 14.2 & -1.4 & 10.1 & 32.3 \\
\hline PBE0AC & 0.5 & -2.9 & 5.4 & -14.3 & -3.2 & 3.5 & -9.5 \\
\hline SCANAC & 9.2 & -11.9 & 15.9 & -24.4 & 3.8 & -8.3 & 4.6 \\
\hline SCANOAC & 8.4 & -10.0 & 13.3 & -21.6 & 3.9 & -8.1 & -2.5 \\
\hline LC-PBETPSS(1.02) ${ }^{\mathrm{b}}$ & 2.9 & -3.0 & 4.7 & -12.9 & 3.6 & -5.3 & 3.8 \\
\hline
\end{tabular}

a) Reference values are from Gaussian type geminal calculations as quoted in Ref. [28]

b) The value of IP optimized range-separation parameter shown in parenthesis

agreement with the reference values. This signifies that the density, density matrix, and response properties (static and frequency-dependent) are correctly described in this functional. Its resulting error of $3.8 \%$ in total interaction energy compares favorably with the previously studied range-separated GGA in Ref. [28] that predicted 5.3\% error in the same basis set.

We conclude that in two-electron systems, and for representative functionals PBE and SCAN, adding metaingredients to GGA is more effective than the hybridization of GGA, in improving the energy components. The quality of dispersion energy in SCAN and SCAN0 in two-electron systems is worth noting. The electrostatic energy improves only upon inclusion of the AC correction. Below, we examine how these conclusions hold-up in SAPT (DFT) for polyelectron monomers.

b. Overall suitability for variety of interaction types

In Table 2, we show the interaction energies of all the complexes in TK21 $\mathrm{S}_{1}$ set and the percentage errors of SAPT (DFT) with respect to SAPT (CCSD) for all considered meta-GGAs. The complexes in TK21 set can be grouped into ion-molecule interactions, hydrogen-bonded systems from typical $\left(\mathrm{H}_{2} \mathrm{O}\right.$ dimer $)$ to unorthodox $\left(\mathrm{C}_{2} \mathrm{H}_{6}\right.$ - $\mathrm{HCN}$, i.e., proton donating to a methyl group), a hydrocarbon dimer $\left(\mathrm{CH}_{4} \mathrm{di}-\right.$ mer), interactions of quadrupolar molecules from weak $\left(\mathrm{N}_{2}\right.$ dimer) to donor-acceptor (PCCP dimer), to typical van der Waals systems (molecule-He and $\mathrm{Ar}_{2}$ ). The objective here is to find if SAPT(DFT) based on meta-GGAs succeeds for any particular type of complexes.

An analog of Table 2 for the $S_{2}$ set of dimers computed in the aug-cc-pVTZ basis set is included in the Supplementary Information as Table $\mathrm{S} 1$.

In ion-molecule interactions, MVS and SCAN perform very well. In this group, the addition of asymptotic correction appears to be of no help. In hydrogen-bonded complexes,
MVS and SCAN are also good performers. AC correction is marginally helpful; the addition of HF-exchange to SCAN is beneficial. Although the range separated HF-exchange in LCPBETPSS appears ineffective even in $\mathrm{H}_{2} \mathrm{O}$ and HF dimers, its effectiveness improves greatly in the larger basis set (see Table S1). It is noteworthy that TPSS and revTPSS perform poorly in this group and the addition of AC still keeps the errors in the double-digit range.

For weakly interacting $\mathrm{CH}_{4}$ dimer, a simplest hydrocarbon interaction, all the functionals except SCANO and LCPBETPSS work poorly. Since SAPT(HF) [9] performs very well for this system, we hypothesize that the problem lies in the quality of DFT exchange at low reduced gradients.

In the acetylene dimer, two structures PD and T are very well reproduced by SCAN and LC-PBETPSS as well as all the AC-corrected SCAN. The S structure is very weakly bound; consequently, even small inaccuracies lead to large double-digit errors. The case of $(\mathrm{NCCN})_{2}$ and $(\mathrm{PCCP})_{2}$ is interesting: Both are donor-acceptor complexes for which SAPT generally works poorly [44]. Nevertheless, for the former, SAPT based on LC-PBETPSS and SCAN0 performs well. The remaining complexes are typical van der Waals systems dominated by dispersion in subtle balance with the repulsive exchange. Again, the errors appear large because the effects are very small. Here, both the AC correction and inclusion of exact exchange bring a significant improvement, and SCANOAC and LC-PBETPSS are the only reliable performers. $\mathrm{Ar}_{2}$ is well reproduced by the majority of functionals, particularly those AC-corrected.

\section{c. Performance for electrostatic energy}

Figure 1 shows relative percent errors from different SAPT(DFT) methods in the form of box-and-whiskers plots; two wave function-based methods SAPT(HF) and SAPT2+ (CCD) are added for comparison. In TPSS and revTPSS, the 
Table 2 Reference SAPT(CCSD) interaction energies (in $\mathrm{mH}$ ) for TK21 molecule set and the percentage errors in SAPT(DFT) using the listed metaGGA functionals. PBE0AC is included for comparison. Basis set is aug-cc-pVDZ

\begin{tabular}{|c|c|c|c|c|c|c|c|c|c|c|c|c|c|}
\hline \multirow[t]{2}{*}{ Complex } & \multirow{2}{*}{$\begin{array}{l}\mathrm{E}_{\text {int }} \\
\mathrm{SAPT} \\
\text { (CCSD) }\end{array}$} & \multicolumn{12}{|c|}{ SAPT (DFT) $\%$ errors } \\
\hline & & $\begin{array}{l}\text { PBE0 } \\
\text { AC }\end{array}$ & TPSS & TPSSAC & revTPSS & $\begin{array}{l}\text { revTPSS } \\
\mathrm{AC}\end{array}$ & MVS & MVSAC & SCAN & SCANAC & SCANO & $\begin{array}{l}\text { SCAN0 } \\
\text { AC }\end{array}$ & $\begin{array}{l}\text { LC- } \\
\text { PBETPSS }\end{array}$ \\
\hline $\mathrm{F}^{-}-\mathrm{HF}$ & -58.98 & -6.5 & 6.9 & 8.8 & 9.9 & 11.7 & -4.9 & -4.3 & 3.8 & 5.0 & -11.5 & -11.2 & -11.5 \\
\hline $\mathrm{F}^{-}-\mathrm{H}_{2} \mathrm{O}$ & -25.59 & -0.5 & 12.7 & 14.2 & 15.0 & 16.4 & -1.8 & -1.3 & 7.8 & 8.7 & -6.8 & -6.5 & 1.1 \\
\hline $\mathrm{Na}^{+}-\mathrm{H}_{2} \mathrm{O}$ & -36.19 & -0.8 & 3.7 & 2.1 & 4.3 & 2.7 & -1.4 & -2.0 & 0.8 & -0.5 & -2.6 & -3.1 & -3.6 \\
\hline HF-HF & -4.78 & -4.4 & 23.4 & 8.2 & 24.4 & 9.5 & -2.5 & -5.5 & 7.7 & 0.6 & -9.2 & -11.3 & -18.8 \\
\hline $\mathrm{H}_{2} \mathrm{O}-\mathrm{H}_{2} \mathrm{O}$ & -5.26 & -1.8 & 23.8 & 12.9 & 25.0 & 14.4 & -5.9 & -5.9 & 9.8 & 6.2 & -7.4 & -7.3 & -10.6 \\
\hline $\mathrm{NH}_{3}-\mathrm{CH}_{4}$ & -0.91 & 16.4 & 26.6 & 23.8 & 28.1 & 26.4 & 3.6 & 16.5 & 14.0 & 21.7 & 4.0 & 18.0 & 11.9 \\
\hline $\mathrm{NH}_{3}-\mathrm{H}_{2} \mathrm{O}$ & -6.92 & 3.4 & 18.5 & 14.8 & 19.4 & 16.0 & -5.1 & -1.0 & 10.6 & 12.0 & -3.5 & -0.1 & -5.4 \\
\hline $\mathrm{C}_{2} \mathrm{H}_{6}-\mathrm{HCN}$ & -1.22 & 3.0 & 21.4 & 16.0 & 24.2 & 19.8 & -1.0 & 8.3 & 9.8 & 13.4 & -3.6 & 3.7 & -1.5 \\
\hline $\mathrm{CH}_{4}-\mathrm{CH}_{4}$ & -0.72 & 24.3 & 22.0 & 24.3 & 21.3 & 24.5 & 16.3 & 29.7 & 17.9 & 28.1 & 11.4 & 26.9 & 14.1 \\
\hline $\mathrm{C}_{2} \mathrm{H}_{2}-\mathrm{C}_{2} \mathrm{H}_{2}-\mathrm{PD}$ & -1.72 & -5.2 & 17.1 & 4.7 & 15.6 & 3.8 & -18.2 & -13.7 & 1.6 & -0.7 & -10.5 & -10.6 & 1.4 \\
\hline $\mathrm{C}_{2} \mathrm{H}_{2}-\mathrm{C}_{2} \mathrm{H}_{2}-\mathrm{S}$ & -0.21 & 26.4 & 55.7 & 16.3 & 56.5 & 18.0 & 77.2 & 71.4 & 58.5 & 38.0 & 55.7 & 44.0 & 64.4 \\
\hline $\mathrm{C}_{2} \mathrm{H}_{2}-\mathrm{C}_{2} \mathrm{H}_{2}-\mathrm{T}$ & -1.74 & -9.8 & 15.2 & 1.1 & 14.3 & 1.0 & -19.8 & -13.9 & -1.7 & -4.4 & -14.2 & -14.3 & -1.8 \\
\hline NCCN-NCCN & -2.73 & 13.8 & 21.1 & 16.1 & 21.1 & 16.4 & 11.3 & 17.0 & 13.8 & 15.3 & 8.1 & 10.9 & 7.0 \\
\hline РССР-РCСР & -3.18 & 16.9 & 25.1 & 22.8 & 24.3 & 22.5 & 14.6 & 25.0 & 21.7 & 26.8 & 16.4 & 20.3 & 32.5 \\
\hline $\mathrm{N}_{2}-\mathrm{N}_{2}$ & -0.38 & 11.3 & 28.0 & 2.9 & 26.8 & 2.9 & 15.3 & 8.3 & 21.8 & 9.8 & 17.0 & 15.4 & 16.3 \\
\hline $\mathrm{P}_{2}-\mathrm{P}_{2}$ & -1.01 & 11.9 & 19.3 & 10.2 & 18.5 & 10.3 & 8.0 & 17.5 & 16.8 & 18.9 & 15.4 & 18.1 & 32.5 \\
\hline $\mathrm{N}_{2} \mathrm{O}-\mathrm{He}(\mathrm{GM})$ & -0.16 & 1.3 & 57.9 & 11.3 & 54.4 & 11.1 & 38.5 & 16.7 & 47.1 & 14.9 & 25.1 & 8.3 & -2.0 \\
\hline $\mathrm{N}_{2} \mathrm{O}-\mathrm{He}(\mathrm{LM})$ & -0.09 & 6.7 & 65.0 & 16.3 & 60.0 & 15.1 & 44.3 & 23.7 & 48.5 & 17.6 & 25.0 & 9.5 & 13.8 \\
\hline $\mathrm{CO}_{2}-\mathrm{He}(\mathrm{GM})$ & -0.10 & 2.9 & 93.1 & 13.3 & 87.1 & 12.7 & 58.3 & 19.1 & 71.4 & 17.1 & 34.1 & 7.5 & -7.5 \\
\hline $\begin{array}{l}\mathrm{CO}_{2}-\mathrm{He}(\mathrm{LM}) \\
\mathrm{Ar}-\mathrm{Ar}\end{array}$ & $\begin{array}{l}-0.07 \\
-0.20\end{array}$ & $\begin{array}{l}4.3 \\
-2.5\end{array}$ & $\begin{array}{l}62.6 \\
24.1\end{array}$ & $\begin{array}{l}7.6 \\
-3.5\end{array}$ & $\begin{array}{l}58.7 \\
23.6\end{array}$ & $\begin{array}{l}7.2 \\
-3.5\end{array}$ & $\begin{array}{l}39.8 \\
7.8\end{array}$ & $\begin{array}{l}17.0 \\
2.7\end{array}$ & $\begin{array}{l}45.6 \\
15.3\end{array}$ & $\begin{array}{l}10.6 \\
1.6\end{array}$ & $\begin{array}{l}21.2 \\
7.0\end{array}$ & $\begin{array}{l}5.6 \\
0.5\end{array}$ & $\begin{array}{l}11.0 \\
5.6\end{array}$ \\
\hline Mean $\%$ error & & 5.3 & 30.6 & 11.6 & 30.1 & 12.3 & 13.1 & 10.7 & 21.1 & 12.4 & 8.2 & 5.9 & 7.1 \\
\hline MAE & & 8.3 & 30.6 & 12.3 & 57.8 & 12.6 & 18.8 & 15.3 & 21.0 & 12.9 & 14.8 & 12.1 & 13.0 \\
\hline
\end{tabular}

electrostatic energy clearly benefits from the inclusion of the $\mathrm{AC}$ correction. These two afford some of the best electrostatic energies overall. By contrast, the changes in electrostatic energies brought by AC in MVS, SCAN, and SCAN0 are unclear.
In the aug-cc-pVDZ basis set, those functionals show little or no improvement upon the GRAC correction. In the larger basis (Fig. 1 right panel), only SCAN improves (we emphasize that aug-cc-pVTZ calculations are performed on a smaller subset of
Fig. 1 Box plots for electrostatic energies of TK21 set in aug-ccpVDZ (aVDZ; left) and aug-ccpVTZ (aVTZ; right). Box includes $50 \%$ of error range, and the whiskers include $95 \%$ and $90 \%$ error range for $\mathrm{aVDZ}$ and $\mathrm{aVTZ}$, respectively; the horizontal bars describe the median, and the dots describe the largest outlier. Errors are with respect to SAPT(CCSD) benchmarks [9]

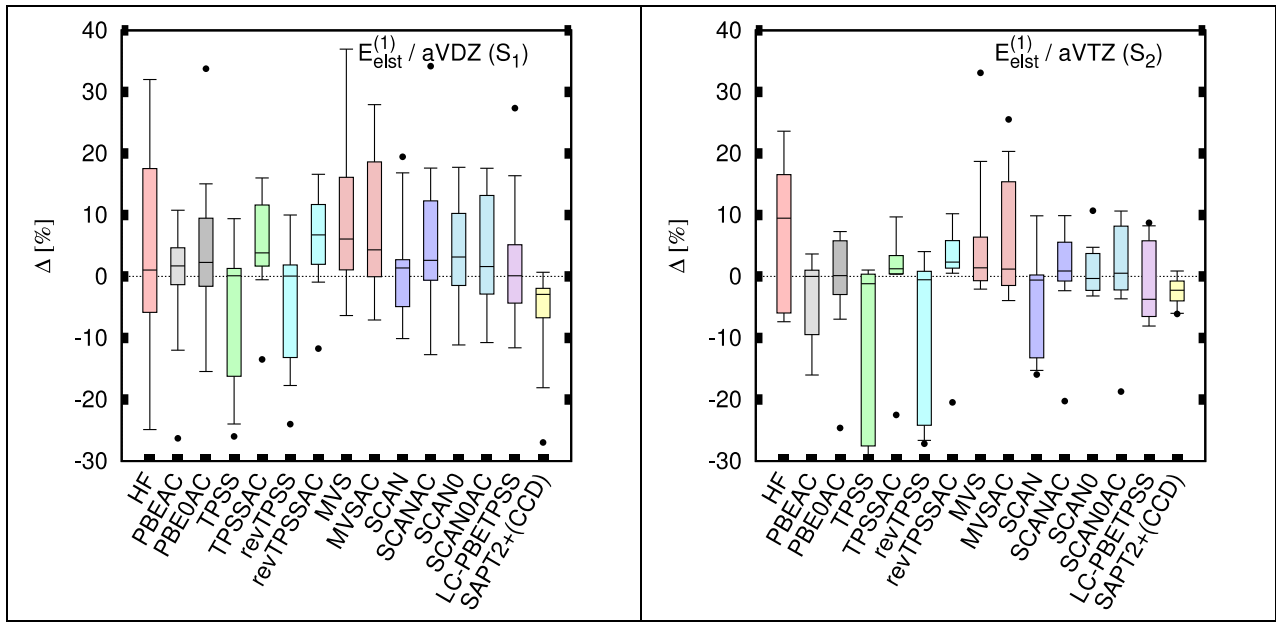


complexes $\mathrm{S}_{2}$ ). Note that even without the AC correction SCAN0 is a reliable performer in both basis sets.

The electrostatic energy is a sensitive measure of the quality of density; from this, we can conclude that most of SCF DFT densities considered here are superior to the HF density, against the claims of the latter's superiority (see, e.g., Ref. [45]).

\section{d. Performance for first-order exchange}

As stated earlier, the first-order exchange presents more of a challenge for SAPT(DFT) than the electrostatic term because it requires the accurate description of the entire density matrix in addition to electron density. Figure 2 shows relative errors in the first-order exchange repulsion. All DFT methods are again superior to HF. The AC correction generally benefits TPSS and revTPSS. These two functionals again yield some of the best exchange terms. SCAN0 is an excellent performer even without AC. MVS, similarly, appears little affected by $\mathrm{AC}$ and its results are poor. Our LC-meta-GGA performs reasonably well. The larger basis set results confirm the trend with MVSAC as an outlier. The wave function SAPT2 performs very well with the exception of one or two outliers, specifically, the P-containing molecules and ethyne dimer in the S configuration (see also Tables S3 and S10 in the Supplemental Information).

e. Induction and exchange induction

Figure 3 presents the errors in the induction and exchangeinduction terms of TK21 set. To reiterate, the induction and dispersion terms (see below) as well as their exchange counterparts are evaluated using coupled KS response theory. This is important because the corresponding uncoupled terms have one order of magnitude larger errors. The plots indicate particularly poor performance of TPSS and revTPSS and their dramatic improvement upon the AC correction. MVS on the other hand shows no improvement. The remaining meta-
GGAs show uniform good performance. The SAPT2+(CCD) results will be discussed below.

\section{f. Dispersion and exchange-dispersion energies}

Figure 4 shows the errors in the second-order dispersion and exchange-dispersion terms. The two TPSS-based functionals afford surprisingly accurate dispersion energies. The previously noted SCAN performance in $\mathrm{He}_{2}$ holds here as well, although based on some error cancelation between an underestimation of both the dispersion attraction and exchange-dispersion repulsion. For exchange-dispersion, the AC-corrected TPSS and revTPSS are excellent. The LC-metaGGA performs very well for both terms, although the spread of errors is larger than in other functionals. MVSAC acts as an outlier in both the dispersion and its exchange counterpart.

The performance of wave function SAPT2 calls for an explanation at this point. The SAPT2 version considered here evaluates the exchange-induction at the coupled level of theory and with partial account of the intrasystem correlation [46]. The large percent errors in the exchange-induction term are being dominated by van der Waals dimers where exchange-induction effects are close to zero. The exchange-dispersion, on the other hand, was computed at the uncoupled level of theory and in the zeroth order with respect to the intrasystem correlation operator. This approximation leads to double-digit percentage errors due to a severe underestimation of this repulsive term. Note that in SAPT(HF) calculations, the exchange-dispersion energy is obtained at the coupled level of theory which leads to slightly better agreement with the SAPT(CCSD) benchmark.

\section{g. Total interaction energy}

In Fig. 5 the errors in total interaction energies of TK21 set are displayed. The first question concerns whether the meta-GGAs
Fig. 2 Box plots for first-order exchange of TK21 molecule set in aug-cc-pVDZ (aVDZ; left) and aug-cc-pVTZ (aVTZ; right). Box includes $50 \%$ of error range, and the whiskers include $95 \%$ and $90 \%$ error range for aVDZ and aVTZ, respectively; the horizontal bars describe the median, and the dots describe the largest outlier. Errors are with respect to SAPT(CCSD) benchmarks [9]

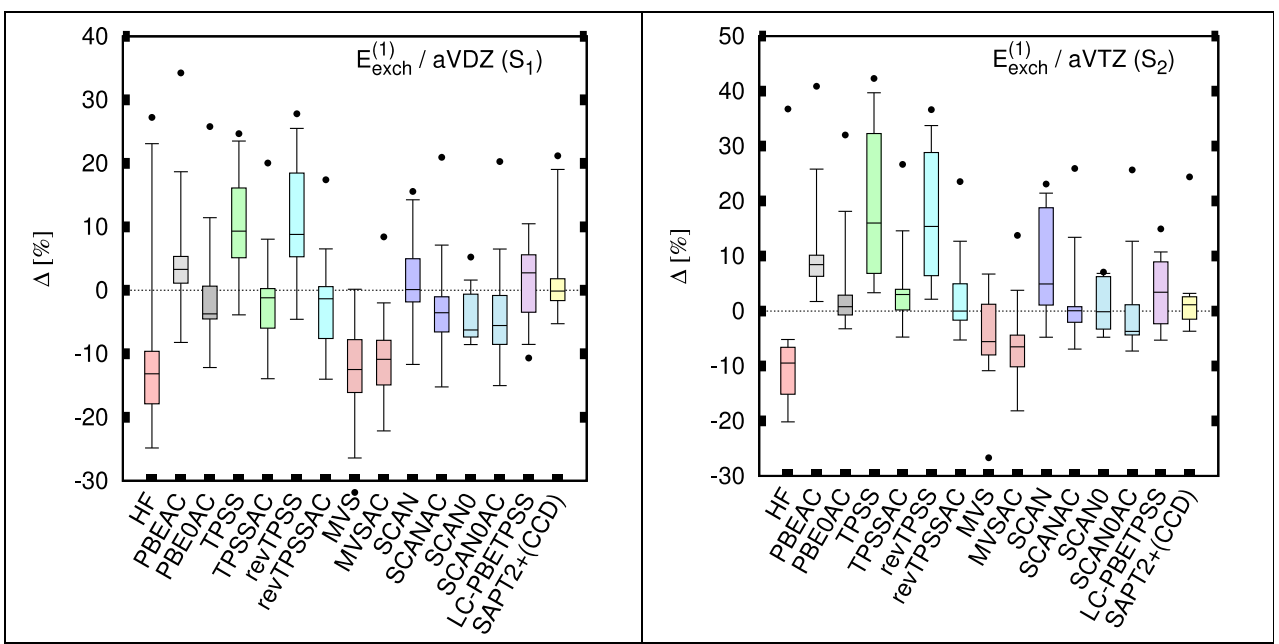


Fig. 3 Box plots for the induction (left) and exchange-induction (right) energies of TK21 set in aug-cc-pVTZ (aVTZ) basis set. Box includes $50 \%$ of error range and the whiskers include $90 \%$ error range; the horizontal bars describe the median, and the dots describe the largest outlier. Errors are with respect to SAPT(CCSD) benchmarks [9]

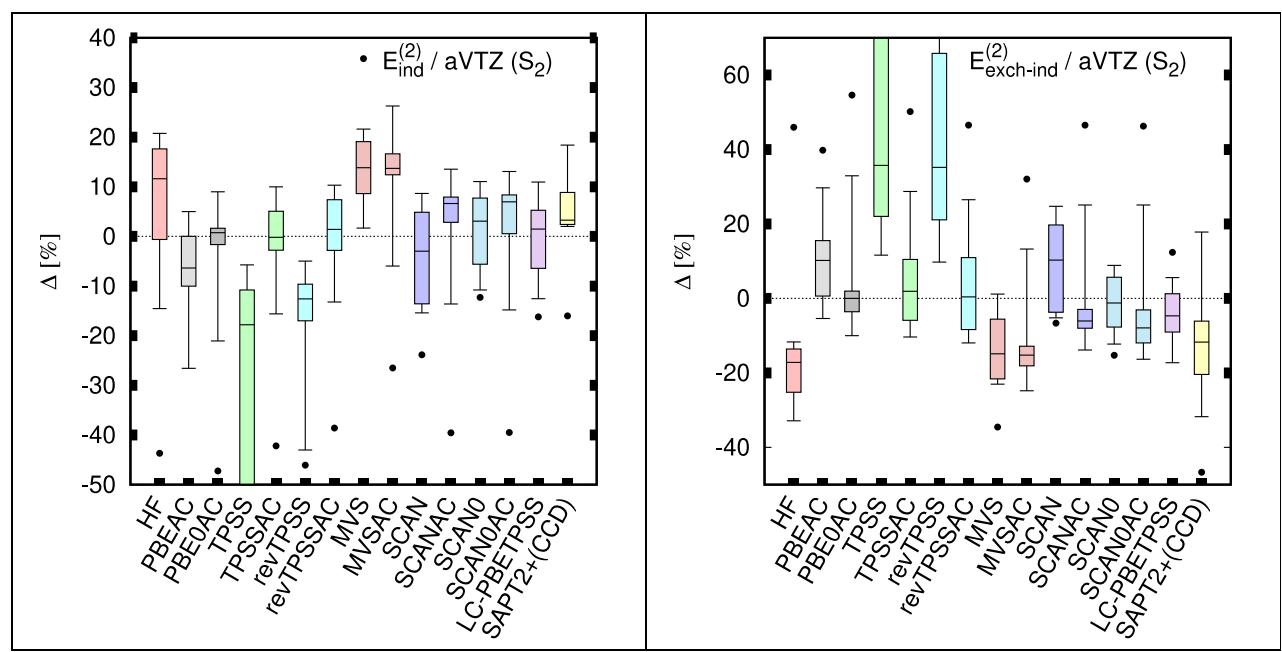

are clearly better than GGAs in SAPT? GGAs are here represented by PBEAC and PBE0AC, two popular choices of SAPT(DFT) calculations. Three non-hybrid, AC-corrected meta-GGA functionals (TPSS, revTPSS, and SCAN) are better than the non-hybrid GGA - PBEAC. Among the hybrids, SCAN0 and LC-meta-GGA come close to PBE0AC but with a larger spread of errors. In the case of LC-PBETPSS, this is in part due to the fact that the range-separation parameters have not been individually tuned for this functional (see Sec. 2.b). Note that interaction energy errors in MVSAC, which was found above to yield poor individual components, are similar to the SCAN0AC case thus indicating a systematic error cancelation in MVS.

The wave function SAPT2+(CCD) is better than any SAPT(DFT) methods, but outliers do exist. It is clear that for the most part, SAPT based on DFT is always superior to that based on HF.

To shed more light on the above performance, we explore how well meta-GGAs satisfy the Koopmans' theorem, the issue critical to the SAPT results [15]. Figure 6 shows the comparison of $\Delta_{\mathrm{XC}}$, that is, the energy value by which the potential needs to be shifted by the AC correction, as computed for TK21 monomers in GGA and meta-GGA and their hybrids. The shown values are with respect to the experimental ionization potentials. Figure 6 demonstrates slight improvements in the theorem's satisfaction between PBE and SCAN and minimal improvement between PBE0 and SCAN0. Much larger changes result from the addition of HF-exchange to both. The data clearly show that even at the most sophisticated hybrid meta-GGA level, the AC correction is still needed to affix the HOMO energy at the correct value.

The AC correction has some negatives. Namely, the $\mathrm{v}_{\mathrm{XC}}$ potential so corrected becomes "stray," i.e., not a functional derivative of any energy formula [47]. For these reasons, one may be interested in finding functionals suitable for SAPT (DFT) without the AC correction. Obviously, LC-corrected functionals serve this purpose. Of the other functionals, SCAN0, while not perfect, could also be useful in SAPT without correcting.
Fig. 4 Box plots for the dispersion (left) and exchangedispersion (right) energies of TK21 set in aug-cc-pVTZ (aVTZ) basis set. Box includes $50 \%$ of error range, and the whiskers include $90 \%$ error range; the horizontal bars describe the median, and the dots describe the largest outlier. Errors are with respect to SAPT(CCSD) benchmarks [9]

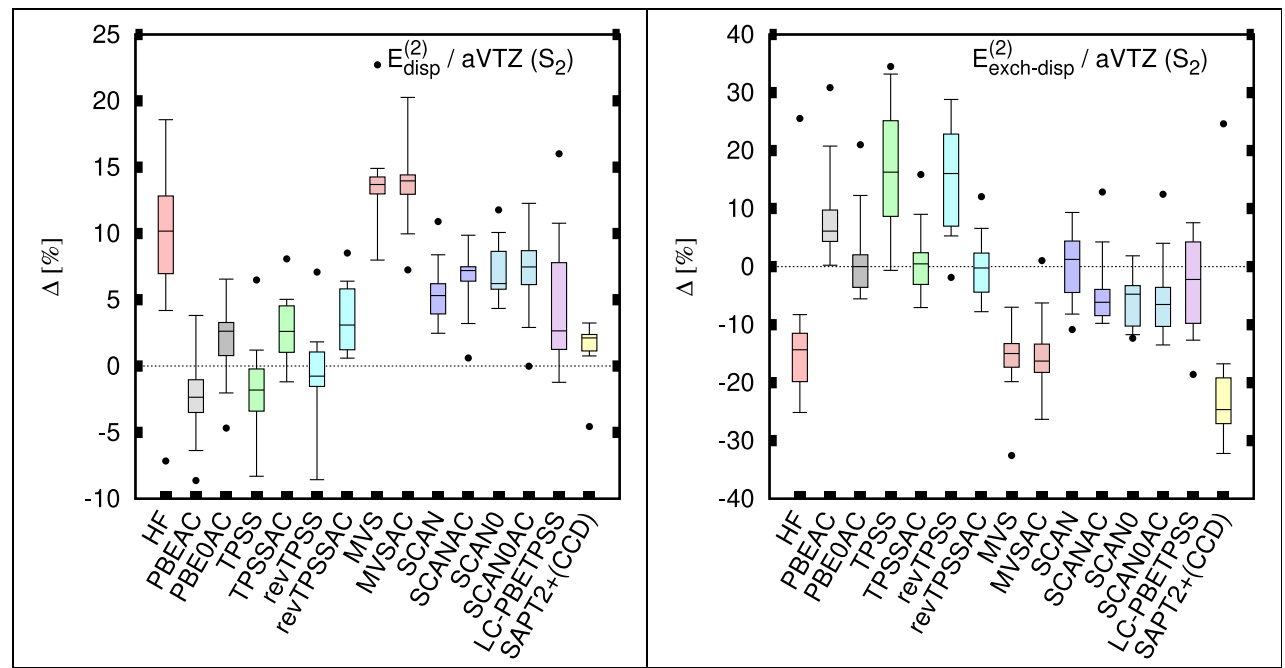


Fig. 5 Box plots for the total interaction energy in in aug-ccpVDZ (aVDZ; left) and aug-ccpVTZ (aVTZ; right). Box includes $50 \%$ of error range, and the whiskers include $95 \%$ and $90 \%$ error range for aVDZ and aVTZ, respectively; the horizontal bars describe the median, and the dots describe the largest outlier. Errors are with respect to SAPT(CCSD) benchmarks [9]

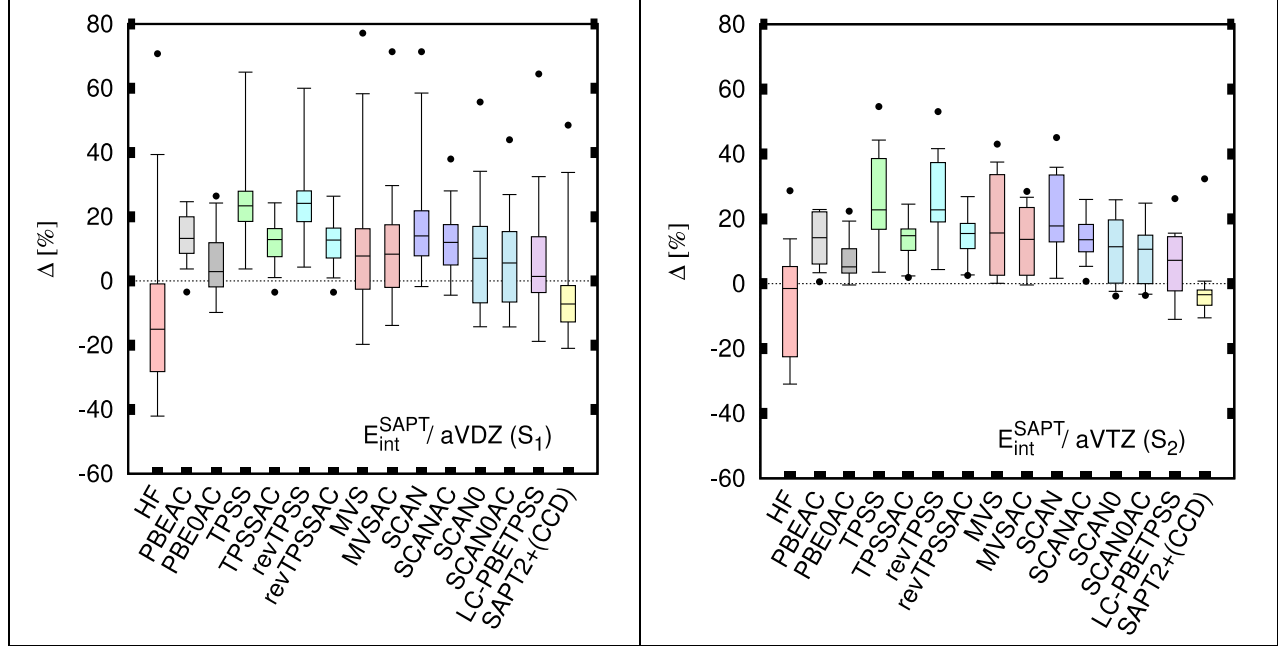

\section{Summary and conclusions}

Meta-GGAs have more sophisticated mathematical forms and hence can satisfy more exact constraints. To what extent satisfying these constraints helps in SAPT(DFT) was our question in this paper. In fact, the constraints apply to the energy formula and their fulfillment is no guarantee that the functional derivative, $\mathbf{v}_{\mathrm{XC}}$, would similarly improve. In SAPT, it is the $\mathrm{v}_{\mathrm{XC}}$ potential which is of primary importance. That is why testing of these new functionals within SAPT is needed.

The correct potential asymptote can be secured in two ways: either by using an asymptotic correction, for example, of the GRAC type, or by employing a long-range correction via the range separation of electron-electron interactions. Examining the compatibility of these AC and LC corrections with the new meta-GGAs is also crucial for SAPT(DFT). Our results indicate that the older meta-GGAs, TPSS and revTPSS, benefit from the GRAC correction since we observe an improvement in total interaction energies as well as

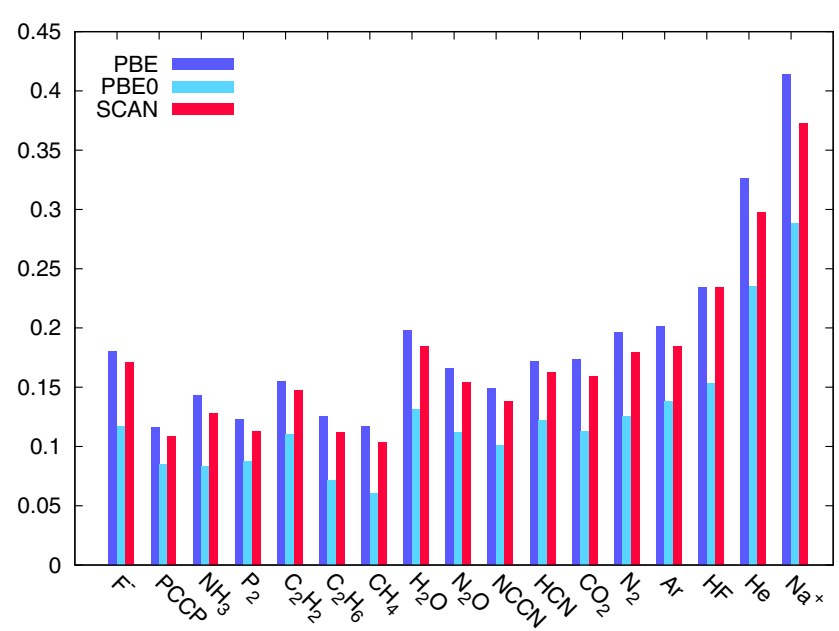

Fig. 6 Potential shift $\Delta_{\mathrm{XC}}=\mathrm{IP}+\mathrm{e}_{\mathrm{HOMO}}$ (in Hartree) for monomers of the TK21 set from aVDZ computation dramatic improvements in the energy components. In the newer meta-GGAs, MVS and SCAN, improvements in individual energy contributions are moderate but effectively add up to a notable reduction of errors in total interaction energies.

It is worth noting that the LC correction is fully compatible with meta-GGAs as our LC-meta-GGA is one of the best methods overall. The results could be further improved by tuning of the range-separation parameters specifically for LC-PBETPSS.

Electrostatic energy can serve as the most sensitive diagnostic tool in assessing electron densities of monomers. The quality of DFT densities has recently become a topic of intense literature debate concerning the DFT's ability of accurately describing the energy and density simultaneously [48]. For both SCAN- and TPSS-based functionals, electrostatic energies agree with reference to within $5 \%$ or better which is an excellent result. The only meta-GGA which does not yield accurate densities is MVS. Also, in all the cases examined here, the DFT densities are superior to HF ones.

The first-order SAPT(DFT) exchange energies, which depend on the quality of the density matrix, appear to be slightly less accurate than electrostatics even upon the AC correction. However, SCAN0 yields some of the best exchange energies in both the first and second orders even without the AC correction.

Which method gives the best values of dispersion energy is also of prime importance to selecting the density functional approximation for SAPT(DFT). TPSS and revTPSS give excellent dispersion energies, provided that the AC correction is applied. The SCAN-based functionals moderately underestimate the dispersion energies leading to the relative errors below $10 \%$ regardless of AC. This is partially compensated by similar underestimation of the exchange-dispersion contribution. MVS is again an outlier with errors much larger in magnitude than the rest of meta-GGAs. 
Our results show that meta-GGAs (with the exception of MVS) represent a definite progress in SAPT(DFT) compared to pure GGA, such as PBEAC, with their more consistent predictions of energy components. However, none of the meta-GGAs is better than the hybrid GGA approach PBE0AC. TPSS and revTPSS with AC come close to PBE0AC in predicting SAPT components, but the errors build up and the total energies are less accurate than those of SAPT(PBE0AC). On the positive note, we find that the only DFT functional which can be used in SAPT without an AC correction is SCAN0 thus providing the evidence of a good quality of both the orbitals and orbital energies. This is a clear improvement with respect to all GGAs studied in the literature. The long-range corrected meta-GGA, LC-PBETPSS, is a very reliable performer both in terms of total interaction energies and all the energy components, thus demonstrating that range-separation is a robust scheme of enforcing the correct asymptote of the exchange-correlation potential.

Acknowledgments The work of MH was supported in part by the Kosciuszko Foundation. He wishes to thank Prof. Dominika Zgid for her hospitality during the Kosciuszko Foundation scholarship.

Open Access This article is licensed under a Creative Commons Attribution 4.0 International License, which permits use, sharing, adaptation, distribution and reproduction in any medium or format, as long as you give appropriate credit to the original author(s) and the source, provide a link to the Creative Commons licence, and indicate if changes were made. The images or other third party material in this article are included in the article's Creative Commons licence, unless indicated otherwise in a credit line to the material. If material is not included in the article's Creative Commons licence and your intended use is not permitted by statutory regulation or exceeds the permitted use, you will need to obtain permission directly from the copyright holder. To view a copy of this licence, visit http://creativecommons.org/licenses/by/4.0/.

\section{References}

1. Jeziorski B, Moszynski R, Szalewicz K (1994) Perturbation theory approach to intermolecular potential energy surfaces of van der Waals complexes. Chem Rev 94:1887-1930

2. Chałasiński G, Szczęśniak MM (1994) Origins of structure and energetics of van der Waals clusters from ab initio calculations. Chem Rev 94:1723-1765

3. Jeziorski B, Szalewicz K, Chałasiński G (1978) Symmetry forcing and convergence properties of perturbation expansions for molecular interactions. Int J Quantum Chem 14:271-287

4. Rybak S, Jeziorski B, Szalewicz K (1991) Many-body symmetryadapted perturbation theory of intermolecular interactions $\mathrm{H}_{2} \mathrm{O}$ and HF dimers. J Chem Phys 95:6576-6601

5. Misquitta AJ, Podeszwa R, Jeziorski B, Szalewicz K (2005) Intermolecular potentials based on symmetry-adapted perturbation theory including dispersion energies from time-dependent density functional calculations. J Chem Phys 123:214103

6. Szalewicz K (2012) Symmetry adapted perturbation theory. WIREs Comput Mol Sci 2:254-272

7. Holzer C, Klopper W (2017) Communication: symmetry-adapted perturbation theory with intermolecular induction and dispersion energies from the Bethe-Salpeter equation. J Chem Phys 147: 181101

8. Boese AD, Jansen G (2019) ZMP-SAPT: DFT-SAPT using ab initio densities. J Chem Phys 150:154101

9. Korona T (2013) A coupled cluster treatment of intramonomer electron correlation within symmetry-adapted perturbation theory: benchmark calculations and a comparison with a density-functional theory description. Mol Phys 111:3705-3715

10. Hapka M, Przybytek M, Pernal K (2019) Second-order dispersion energy based on multireference description of monomers. J Chem Theory Comput 15:1016-1027

11. Hapka M, Przybytek M, Pernal K (2019) Second-order exchangedispersion energy based on multireference description of monomers. J Chem Theory Comput 15:6712-6723

12. Misquitta AJ, Szalewicz K (2002) Intermolecular forces from asymptotically corrected density functional description of monomers. Chem Phys Lett 357:301-306

13. Misquitta AJ, Jeziorski B, Szalewicz K (2003) Dispersion energy from density-functional theory description of monomers. Phys Rev Lett 91:033201

14. Misquitta AJ, Szalewicz K (2005) Symmetry-adapted perturbationtheory calculations of intermolecular forces employing densityfunctional description of monomers. J Chem Phys 122:214109

15. Heßelmann A, Jansen G (2002) First-order intermolecular interaction energies from Kohn-sham orbitals. Chem Phys Lett 2002(357):464-470

16. Heßelmann A, Jansen G (2002) Intermolecular induction and exchange-induction energies from coupled-perturbed Kohn-sham density functional theory. Chem Phys Lett 362:319-325

17. Heßelmann A, Jansen G (2003) Intermolecular dispersion energies from time-dependent density functional theory. Chem Phys Lett 367:778-784

18. Jansen G (2014) Symmetry-Adapted Perturbation Theory Based on Density Functional Theory for Noncovalent Interactions. WIREs Comput Mol Sci 4:127-144

19. Perdew JP, Schmidt K (2001) Jacob's ladder of density functional approximations for the exchange-correlation energy. AIP Conf Proc 577:1

20. Zhao Y, Schultz NE, Truhlar DG (2005) Exchange-correlation functional with broad accuracy for metallic and nonmetallic compounds, kinetics, and noncovalent interactions. J Chem Phys 123: 161103

21. Zhao Y, Truhlar DG (2008) The M06 suite of density functionals for main group thermochemistry, thermochemical kinetics, noncovalent interactions, excited states, and transition elements: two new functionals and systematic testing of four M06-class functionals and 12 other functionals. Theor Chem Accounts 120:215241

22. Sun J, Ruzsinszky A, Perdew JP (2015) Strongly constrained and appropriately normed semilocal density functional. Phys Rev Lett 115:036402

23. Sun J, Ruzsinszky A, Perdew JP (2015) Semilocal density functional obeying a strongly tightened bound for exchange. Proc Natl Acad Sci U S A 112:685-689

24. Becke AD, Roussel MR (1989) Exchange holes in inhomogeneous systems: a coordinate-space model. Phys Rev A 39:3761-3767

25. Dunning Jr TH (1989) Gaussian basis sets for use in correlated molecular calculations. I. The atoms boron through neon and hydrogen. J Chem Phys 90:1007-1023

26. Kendall RA, Dunning Jr TH, Harrison RJ (1992) Electron affinities of the first-row atoms revisited. Systematic basis sets and wave functions. J Chem Phys 96:6796-6806

27. Cencek W, Szalewicz K (2013) On asymptotic behavior of density functional theory. J Chem Phys 139:024104

28. Cencek W, Szalewicz K (2014) Erratum: on asymptotic behavior of density functional theory. J Chem Phys 140:149902 
29. Tao J, Perdew JP, Staroverov VN, Scuseria GE (2003) Climbing the density functional ladder: nonempirical meta-generalized gradient approximation designed for molecules and solids. Phys Rev Lett 91:146401

30. Perdew JP, Ruzsinszky A, Csonka GI, Constantin LA, Sun J (2009) Workhorse semilocal density functional for condensed matter physics and quantum chemistry. Phys Rev Lett 103:026403

31. Hui K, Chai JD (2016) SCAN-based hybrid and double-hybrid density functionals from models without fitted parameters. J Chem Phys 144:044114

32. Modrzejewski M, Chałasiński G, Szczęśniak MM (2014) Rangeseparated meta-GGA functional designed for noncovalent interactions. J Chem Theory Comput 10:4297-4306

33. Hapka M, Rajchel Ł, Modrzejewski M, Chałasiński G, Szczęśniak MM (2014) Tuned range-separated hybrid functionals in the symmetry-adapted perturbation theory. J Chem Phys 141:134120

34. Vydrov OA, Scuseria GE (2006) Assessment of a long-range corrected hybrid functional. J Chem Phys 125:234109

35. Gruning M, Gritsenko OV, van Gisbergen SJA, Baerends EJ (2001) Shape corrections to exchange-correlation potentials by gradientregulated seamless connection of model potentials for inner and outer region. J Chem Phys 114:652-660

36. Bast R, Hesselmann A, Sałek P, Helgaker T, Saue T (2008) Static and frequency-dependent dipole-dipole polarizabilities of all closed-shell atoms up to radium: a four-component relativistic DFT study. ChemPhysChem 9:445-453

37. Werner H-J, Knowles PJ, Knizia G, Manby FR, Schütz M (2012) Molpro: a general-purpose quantum chemistry program package. WIREs Comput Mol Sci 2:242-253

38. Mura ME, Knowles PJ (1996) Improved radial grids for quadrature in molecular density-functional calculations. J Chem Phys 104: 9848-9858

39. Hohenstein EG, Sherrill CD (2010) Density fitting of intramonomer correlation effects in symmetry-adapted perturbation theory. $\mathrm{J}$ Chem Phys 133:014101

40. Williams HL, Szalewicz K, Moszynski R, Jeziorski B (1995) Dispersion energy in the coupled pair approximation with noniterative inclusion of single and triple excitations. J Chem Phys 103:4586-4599

41. Patkowski K, Szalewicz K, Jeziorski B (2006) Third-order interactions in symmetry-adapted perturbation theory. J Chem Phys 125: 154107

42. Parrish RM, Burns LA, Smith DGA, Simmonett AC, DePrince III AE, Hohenstein EG, Bozkaya U, Sokolov AY, Di Remigio R, Richard RM, Gonthier JF, James AM, McAlexander HR, Kumar A, Saitow M, Wang X, Pritchard BP, Verma P, Schaefer III HF, Patkowski K, King RA, Valeev EF, Evangelista FA, Turney JM, Crawford TD, Sherrill CD (2017) Psi4 1.1: an open-source electronic structure program emphasizing automation, advanced libraries, and interoperability. J Chem Theory Comput 13:3185-3197

43. Parrish RM, Hohenstein EG, Sherrill CD (2013) Tractability gains in symmetry-adapted perturbation theory including coupled double excitations: $\mathrm{CCD}+\mathrm{ST}(\mathrm{CCD})$ dispersion with natural orbital truncations. J Chem Phys 139:174102

44. Modrzejewski M, Rajchel L, Szczęśniak MM, Chałasiński G (2012) Dispersion-free component of non-covalent interaction via mutual polarization of fragment densities. J Chem Phys 136: 204109

45. Wasserman A, Nafziger J, Jiang K, Kim MC, Sim E, Burke K (2017) The importance of being inconsistent. Annu Rev Phys Chem 68:555-581

46. Hohenstein EG, Sherrill CD (2012) Wavefunction methods for noncovalent interactions. WIREs Comput Mol Sci 2:304-326

47. Gaiduk AP, Staroverov VN (2009) How to tell when a model Kohn-sham potential is not a functional derivative. J Chem Phys 131:044107

48. Medvedev MG, Bushmarinov IS, Sun J, Perdew JP, Lyssenko KA (2017) Density functional theory is straying from the path toward the exact functional. Science 355:49-52

Publisher's note Springer Nature remains neutral with regard to jurisdictional claims in published maps and institutional affiliations. 